\title{
Biologically inspired acoustic sensors: from insect ears to miniature microphones
}

\author{
James Windmill
}

University of Strathclyde, United Kingdom

Taking inspiration from insect ears to develop an acoustic device is not new. Research to take inspiration from the highly directional, sub-wavelength, Ormia ochracea fly's ear to produce miniature directional microphones is well-known. Since the 1990s, researchers have tried to implement Ormia based micro-electro-mechanical systems devices, typically using standard silicon or similar microfabrication. Much of this time has been spent trying to circumvent the fact that Ormia evolved to hear one specific frequency, that of a calling cricket, and so is not a broadband system as you would require for an audio microphone. Further, building a silicon system inspired by the mechanics of insect ears leads to various compromises. This talk will discuss ongoing work at the University of Strathclyde on silicon microfabrication of acoustic devices inspired by the Ormia system. It will focus on efforts to create MEMS microphones designed with multiple resonance frequencies in the human vocal range. It will also describe ongoing research into the use of active feedback in MEMS acoustic systems, inspired by auditory hearing mechanisms. Finally, it will also look at the latest research by the Strathclyde team into the application of 3D microfabrication for the development of bio-inspired acoustic devices.

Accepted manuscript of the following research output: Windmill, James. / Biologically inspired acoustic sensors : from insect ears to miniature microphones. In: Journal of the Acoustical Society of America. 2018 ; Vol. 143, No. 3. 